\title{
Dynamic Feathering: Minimising Blending Artefacts in View-Dependent Rendering
}

\author{
J.-Y. Guillemaut, J. Kilner, J. Starck, A. Hilton \\ Centre for Vision, Speech, and Signal Processing, University of Surrey, U.K. \\ \{J.Guillemaut, J.Kilner, J.Starck, A.Hilton\} @surrey.ac.uk
}

Keywords: Image-based rendering, view-dependent texture mapping, feathering, image blending, image compositing.

\begin{abstract}
Conventional view-dependent texture mapping techniques produce composite images by blending subsets of input images, weighted according to their relative influence at the rendering viewpoint, over regions where the views overlap. Geometric or camera calibration errors often result in a loss of detail due to blurring or double exposure artefacts which tends to be exacerbated by the number of blending views considered. We propose a novel view-dependent rendering technique which optimises the blend region dynamically at rendering time, and reduces the adverse effects of camera calibration or geometric errors otherwise observed. The technique has been successfully integrated in a rendering pipeline which operates at interactive frame rates. Improvement over state-of-the-art view-dependent texture mapping techniques are illustrated on a synthetic scene as well as real imagery of a large scale outdoor scene where large camera calibration and geometric errors are present.
\end{abstract}

\section{Introduction}

Realistic rendering of a scene from an arbitrary viewpoint is a challenging task which has been approached from two fundamentally different perspectives. The first approach, called model-based rendering, derives a single 3D model together with its surface appearance properties (reflectance, albedo, texture, material...) and requires explicit modelling of such properties, which can be an extremely complex task depending on the nature of the scene. In contrast, the second approach, called image-based rendering [8, 12], replaces the explicit model by a simpler implicit representation where the scene is described as a large collection of images captured by a moving camera. There exist also hybrid techniques which combine the advantages of the two previous approaches by using an approximate scene model, often called a geometric proxy, to interpolate the appearance from a relatively smaller number of camera images compared to pure image-based techniques. A review of these techniques can be found in [19, 23].

In conventional image-based rendering a single texture is derived by maximising the surface sampling rate in the camera images when transferring appearance from the cameras to texture domain. This introduces visible seams in the texture domain which can be blended using non-linear techniques (e.g. multi-resolution spline) to preserve texture detail. Viewdependent texture mapping techniques on the other hand can maximise surface sampling only by weighting the relative influence of the different cameras. A blend is then performed across complete surface regions where there is an overlap between views. Such a view-dependent blending can lead to a loss of detail, particularly in the presence of geometric errors or inaccurate camera calibration which lead to blurring or double exposure artefacts (such artefacts are visible in the first two columns of Fig. 4 and Fig. 6).

This paper introduces a hybrid approach to view-dependent rendering that automatically generates a view-dependent blend region in texturing from multiple views. In this approach, the scene is represented as a collection of images together with their depth maps. The key idea of the method is to constrain blending to a region in the rendered view that reduces the effects of geometric and camera calibration errors during rendering, while still ensuring a smooth blend of the input images. The technique proposed operates by optimising for each rendering viewpoint the spatial characteristics of the blending area and by defining smooth blending functions of the input images across the computed optimised area. Our contributions are the following. Firstly we propose an algorithm, that we call "dynamic feathering", for performing on-the-fly optimisation of the blending area, and which achieves high quality smooth transitions with minimum image blur in spite of camera calibration errors or inaccurate geometry. Secondly, we describe an implementation of the proposed algorithm into a rendering pipeline which runs at interactive frame rate on a standard PC.

The paper is organised as follows. We start by reviewing existing view-dependent rendering techniques and show their limitations. In Section 3, we describe the proposed dynamic feathering algorithm, starting with the description in the simpler two-view case and then generalising it to an arbitrary number of views. We then describe a full rendering pipeline which incorporates the proposed algorithm. Implementation details for each part of the pipeline are given in Section 5, covering aspects such as efficiency and hardware acceleration. In Section 6.2, results are given first in the case of a simple synthetic example, and then on a complex large-scale outdoor scene. In both cases, inaccurate camera calibration and scene geometry are considered. Finally we conclude and discuss avenues for future work. 


\section{Background}

In the Façade system described in [5], Debevec et al. showed that an impressive degree of photorealism can be achieved by selecting at rendering time the views that are combined according to the rendering viewpoint. The method, called view-dependent texture-mapping, assigns a composite colour at each pixel in the rendered view. The colour is defined as a weighted average of the colours seen by the input views with the smallest angular deviation from the rendered view. The blending weights defined are inversely proportional to the angle magnitudes, and are computed at the centre of every primitive in the geometric proxy. Seams at image projection boundaries are eliminated by feathering all projected images at their boundary by attenuation of the pixel weights using a ramp-shaped transition. An optimised version of the algorithm with real-time capabilities was demonstrated in [4].

Since Debevec's seminal paper, a variety of improvements on the method have been proposed. In [17], Pulli et al. apply the concept of view-dependent texture mapping to a collection of view-dependent textured triangular meshes acquired with an active stereo system. They generate novel views by blending triplets of images whose direction form the smallest angles with the direction of the rendering camera. The blending field at each pixel is defined as the product of three weights which represent for each input camera: i) the proximity to the rendering camera expressed in terms of its barycentric coordinates, ii) the surface sampling based on the mesh's triangle orientations, iii) a feathering weight proportional to the truncated linear distance to the object boundary. Soft $z$ buffering is used to estimate surface visibility.

In [6], Evers-Senne and Koch present a system which generates consistent view-dependent local geometry on the fly from a collection of inaccurate depth maps recorded by a hand-held multi-camera system. The algorithm enforces consistency of the geometric proxies which would otherwise be inconsistent and affect rendering quality due to existing camera calibration errors. Interactive frame rate is demonstrated on complex scenes. In [18], Raskar and Low showed that conventional view-dependent texture mapping approaches which derive blending weights from local information only do not normally produce smooth transitions in the presence of existing depth discontinuities. They tackle the problem by defining global visibility and occlusion constraints, computing for each input image a map of distances to the nearest image boundary or depth discontinuity, which are then used to weight each pixel in the rendered image.

In [2], the blending weights for the $k$-nearest cameras are defined as the sum of three components derived from measures of i) angular distance to the rendering camera, ii) camera resolution and iii) field-of-view. Real-time rendering is achieved by restricting the estimation of the blending weights to a sparse set of uniformly sampled points, from which a dense blending field is then interpolated. In $[22,21]$, the uniform sampling of the blending field is replaced by a non-uniform sampling, where sampling density increases with surface curvature, thus enhancing blending quality. In [13], Pajarola et al. use adaptive depth-image meshing to reduce the complexity of the representation and thereby speed-up rendering time. Hardware acceleration is used to efficiently compute per pixel blending weights for each input image, first rendering separately each input image using a soft $z$-buffer algorithm, then combining the separate outputs using alpha blending of quadrilaterals texture mapped with these images. Per pixel normalisation operations are done directly in hardware using an OpenGL Texture Shader extension.

In [15], the authors use a two pass method for real-time rendering of scenes containing large numbers of triangles. During the first pass they reconstruct the scene surface for cameras located on a predefined camera path, approximating depth variations at each pixel by a piece-wise linear function. During the second pass, the scene is rendered in real-time using the pre-computed depth values. The main drawback of the method is that the camera is restricted to locations along a predefined path. In [16], mesh simplification is used to produce a triangular mesh per input camera. Their rendering pipeline combines up to three input views by assigning at each pixel the colour seen by the closest camera passing the visibility test, visibility being computed directly in the fragment shader. The approach has the advantage of eliminating blurring artefacts since no blending is performed. However, although this may be acceptable for synthetic images, it is likely to produce visible seams in the case of inaccurately calibrated real imagery.

Finally, there exists more sophisticated image compositing techniques such as multi-resolution blending or Poisson blending [14]. A recent survey on such techniques can be found in [20]. These techniques are able to achieve high quality image blend, however they are usually computationally more expensive than the previous techniques and therefore less suitable for real-time view-dependent rendering.

\subsection{Limitations of previous approaches}

The previous approaches present the following limitations. Feather location and dimensions are view-independent, and most often restricted to feathering at an image boundary such as for example in [5, 17]. View-independent feathering weights do not take into account local surface orientation and foreshortening effects, and may thus result in smaller feathers or no feather at all where the surface is slanted. More importantly, view-independent feathering does not take into account scene visibility, which often leads to discontinuous transitions caused by depth discontinuities. An exception is [18] which introduces additional global image constraints to explicitly consider the problem of scene visibility. In addition, existing feathering algorithms blend textures across regions corresponding to the overlap of the different views, which often result in large blending areas and leads to a loss of detail (blurring, double exposure artefacts) if camera calibration or geometry are inaccurate. Such artefacts tend to be exacerbated by the number of blending views combined. These artefacts 
are illustrated in the first two columns of Fig. 4 and Fig. 6.

Our technique, in contrast computes a view-dependent feathering region which minimises the amount of overlap while still allowing smooth transitions. Also because our technique reasons at the image level instead of at local pixel level, better treatment of scene visibility is possible.

\section{Dynamic feathering method}

For clarity, after the problem formulation and general method description, the algorithm is described in the simpler case of two views and then generalised to $N$ views. Implementation details are postponed to the next section.

\subsection{Problem formulation and notations}

Let us consider a scene represented by a collection of $N$ surfaces $\left\{\mathcal{S}_{i}\right\}_{1 \leq i \leq N}$ together with their respective texture images $\left\{\mathcal{T}_{i}\right\}_{1 \leq i \leq N}$ captured from distinct viewpoints $\left\{\boldsymbol{C}_{i}\right\}_{1 \leq i \leq N}$. Each texture $\mathcal{T}_{i}$ can be mapped to the corresponding surface $\mathcal{S}_{i}$ and rendered from a desired viewpoint $\boldsymbol{C}$. We denote by $\left\{\mathcal{I}_{i}\right\}_{1 \leq i \leq N}$ the set of such obtained images. We assumed that only visible parts of the scene are rendered; that is occluded parts have been discarded (we will come back to the implementation details in Section 5). We also assume that a set of view-dependent image scores $\left\{s_{i}\right\}_{1 \leq i \leq N}$ is available. Each score $s_{i}$ reflects the importance that should be given to image $i$ for rendering the current viewpoint. Such scores could be defined in terms of the magnitude of the angle between camera $i$ and the rendering camera by computing for example the inverse of this angle (see [5]) or its cosine (see [17]). More sophisticated measures which take into account the Euclidian distance between image centres (see $[6,13]$ ), or even the camera resolution and field-of-view (see $[2,21]$ ) could also be considered. This choice is independent of the proposed feathering algorithm.

The problem consists in computing the blending field, that is the contribution of each image at each pixel in the view to render. We denote by $w_{f}(\boldsymbol{p}, i)$ the feathering weight for camera $i$ at pixel $\boldsymbol{p}$. Once these weights have been computed the separately rendered images $\left\{\mathcal{I}_{i}\right\}_{1<i<N}$ can be composited using the blending weights $w_{f}(\boldsymbol{p}, i)$ and possibly additional pixel dependent weights $w_{x}(\boldsymbol{p}, i)$. Denoting by $\mathcal{I}_{i}(\boldsymbol{p})$ the colour of pixel $\boldsymbol{p}$ in image $\mathrm{i}$, the resulting composite view $\mathcal{I}$ is such that the colour of pixel $p$ is:

$$
\mathcal{I}(\boldsymbol{p})=\sum_{i=1}^{N} \mathcal{I}_{i}(\boldsymbol{p}) w_{f}(\boldsymbol{p}, i) w_{x}(\boldsymbol{p}, i)
$$

The problem can now be formulated in terms of estimating the set of coefficients $w_{f}(\boldsymbol{p}, i)$ for each camera at each pixel.

Before we describe the dynamic feathering algorithm, let us introduce additional notation. We denote by $\boldsymbol{A}_{i}$ the set of pixels in the rendered view that can be textured only from camera $i$, and by $\boldsymbol{B}$ the set of pixels for which several textures overlap. $\boldsymbol{A}_{i}$ together with $\boldsymbol{B}$ form a partition of the set of all foreground pixels. The computation of the blending weights is trivial in the case of any pixel belonging to $\boldsymbol{A}_{i}$, and we have:

$$
\forall \boldsymbol{p} \in \boldsymbol{A}_{i}, \quad w_{f}(\boldsymbol{p}, j)=\left\{\begin{array}{lll}
1 & \text { if } j=i, \\
0 & \text { if } j \neq i .
\end{array}\right.
$$

The problem of computing the feather can therefore be restricted to pixels belonging to $\boldsymbol{B}$. We denote by $d(\boldsymbol{p}, \boldsymbol{X})$ the distance between a pixel $\boldsymbol{p}$ and the closest pixel in the set $\boldsymbol{X}$.

\subsection{Description of the method}

In order to produce seamless spatial and temporal transitions, we impose the following constraints on the feathering weight functions $w_{f}(\boldsymbol{p}, i)$. Firstly, for any camera $i$, we would like $w_{f}(\boldsymbol{p}, i)$ to be a smooth function with respect to $\boldsymbol{p}$. Note that this constraint is usually not satisfied in conventional viewdependent texture mapping algorithms even if the contributions are forced to fall to zero at image boundaries. The reasons for the violation of this constraint are that i) discontinuities may occur within the interior of an image due for example to an occlusion or a depth discontinuity, ii) the $k$-nearest selection algorithm may select to use only part of an image thus producing a seam at the cut. Secondly, if the rendering camera is close to one the input cameras, we would like $B$ to be textured only with this input camera. This constraint is usually not satisfied by conventional view-dependent texture mapping algorithms which blend a fixed number of best views. In the list of desirable goals of an image-based rendering system given in [2], these two constraints were called the continuity goal and the epipole consistency goal.

In the rendered image, the feather can be characterised for each input view $i$ by: i) the line defining its centre, ii) its width which determines the extent of the feather, and iii) a function defining weight variation when moving away from the centre. We assume that feathers are symmetric and follow a linear variation model in which weights fall off or increase continuously with respect to the distance from the boundary, depending on which side of the feather is considered. Detailed weight computation is now given.

\subsubsection{Two-view case}

Let us start with the simpler case where only two views are blended. The feather centre is defined as the level-set of the function $f: \boldsymbol{p} \rightarrow d\left(\boldsymbol{p}, \boldsymbol{A}_{1}\right) / d\left(\boldsymbol{p}, \boldsymbol{A}_{2}\right)$ for the value $s_{1} / s_{2}$, i.e. the set of pixels $\boldsymbol{p}$ belonging to $\boldsymbol{B}$ which satisfy:

$$
\frac{d\left(\boldsymbol{p}, \boldsymbol{A}_{1}\right)}{s_{1}}=\frac{d\left(\boldsymbol{p}, \boldsymbol{A}_{2}\right)}{s_{2}} .
$$

Note that the image scores are assumed to be strictly positive therefore $s_{1}>0$ and $s_{2}>0$. In the particular case where $s_{1}=s_{2}$, the feather centre is the set of points equidistant from $\boldsymbol{A}_{1}$ and $\boldsymbol{A}_{2}$. This is in agreement with the expectation that both images should contribute equally if they have the same score. On the other hand, if one score is much smaller than the other, for example $s_{1}=\epsilon s_{2}$ with $\epsilon \approx 0$, the feather centre is the 
locus of points satisfying $d\left(\boldsymbol{p}, \boldsymbol{A}_{1}\right)=\epsilon d\left(\boldsymbol{p}, \boldsymbol{A}_{2}\right)$, this set of points tends towards the boundary between $\boldsymbol{A}_{1}$ and $\boldsymbol{B}$ so that $\boldsymbol{B}$ is textured almost solely with camera 2 when $\epsilon$ tends towards zero. This is again compatible with the expectation that mainly the texture with largest score should contribute in this case. If the rendering viewpoint moves continuously from camera 2 to camera 1 , the ratio $s_{1} / s_{2}$ is expected to vary continuously from 0 to infinity, which will result in the feather centre to "slide" from the boundary between $\boldsymbol{A}_{2}$ and $\boldsymbol{B}$ to the boundary between $\boldsymbol{A}_{1}$ and $\boldsymbol{B}$. In Fig. 1, the feather centre is represented as the black dash-dot line, it corresponds to assignments of blending weights of 0.5 in both views.

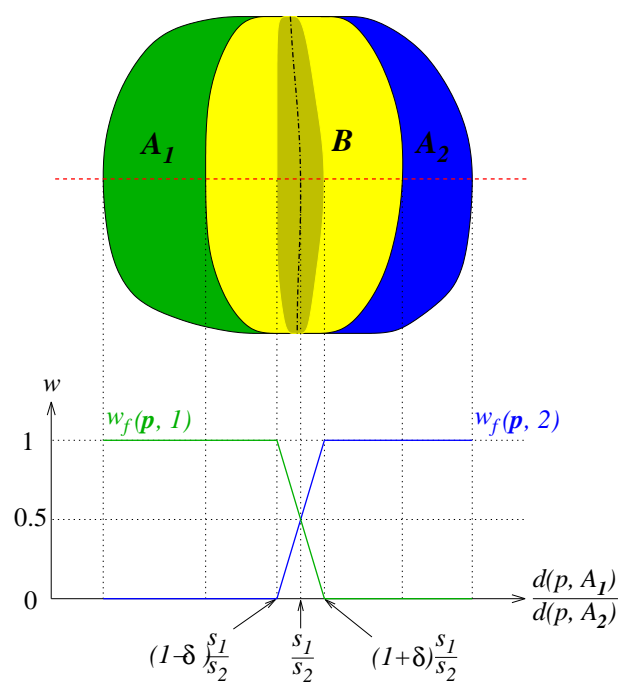

Figure 1: Illustration of feathering weights variations in the two-view case. The top part of the figure shows the visible regions for each views. The feather, whose centre is marked with the dash-dot line, is represented with a darker shade of yellow. The bottom graph illustrates, for each input view, the weight variations along the dashed red line cutting the top figure across.

Now that we have established how to compute the feather centre location, it remains to establish how the feather weights vary when moving away from its centre. We could have defined the width in terms of an absolute number of pixels. However this is problematic because of the variations in the dimensions of the overlap regions. In particular, it is necessary to guarantee that the feather has fallen off completely to zero or increased completely to one within $\boldsymbol{B}$. We achieve this by using a relative feather width $\delta$ (with $0 \leq \delta \leq 1$ ) such that the feather boundaries extremities correspond to the level sets for the function $f$ previously defined for the values $(1-\delta) s_{1} / s_{2}$ and $(1+\delta) s_{1} / s_{2}$ respectively. The resulting weight function $w_{f}(\boldsymbol{p}, 2)$ is a piecewise linear function with respect to the ratio $r_{12}=d\left(\boldsymbol{p}, \boldsymbol{A}_{1}\right) / d\left(\boldsymbol{p}, \boldsymbol{A}_{2}\right)$, which can be written explicitly as:

$w_{f}(\boldsymbol{p}, 2)=$

$$
\left\{\begin{array}{cll}
0 & \text { if } & r_{12} \leq(1-\delta) \frac{s_{1}}{s_{2}}, \\
\frac{1}{2 \delta} \frac{s_{2}}{s_{1}} r_{12}+\frac{\delta-1}{2 \delta .} & \text { if } \quad(1-\delta) \frac{s_{1}}{s_{2}}<r_{12}<(1+\delta) \frac{s_{1}}{s_{2}} \\
1 & \text { if } & r_{12} \geq(1+\delta) \frac{s_{1}}{s_{2}} .
\end{array}\right.
$$

and similarly for $w_{f}(\boldsymbol{p}, 1)$ by permutation of the indices 1 and 2 in the previous expression. The shape of the functions $w_{f}(\boldsymbol{p}, 1)$ and $w_{f}(\boldsymbol{p}, 2)$ is illustrated in Fig. 1.

\subsubsection{Generalisation to $N$ views}

In order to generalise the algorithm to $N$ views, the set of pixels within the overlap region is divided into disjoint regions in each of which the previously described pairwise blending strategy can be applied separately. Let us denote by $\boldsymbol{B}_{i j}$ the set of pixels in $\boldsymbol{B}$ such that for all $k$ such that $1 \leq k \leq N, d\left(\boldsymbol{p}, \boldsymbol{A}_{k}\right) / s_{k}$ is no smaller than $d\left(\boldsymbol{p}, \boldsymbol{A}_{i}\right) / s_{i}$ and $d\left(\boldsymbol{p}, \boldsymbol{A}_{j}\right) / s_{j}$. The set of $\boldsymbol{B}_{i j}$ for all pairs $(i, j)$ forms a partition of $\boldsymbol{B}$ (see Fig. 2). The pairwise algorithm is applied to each set $\boldsymbol{B}_{i j}$ separately. For a given set $\boldsymbol{B}_{i j}$, only images $i$ and $j$ contribute, and their weights are computed according to Eq. (4) after replacement of the indices 0 and $\mathrm{i}$ by $i$ and $j$; the weights for all other images are set to zero.

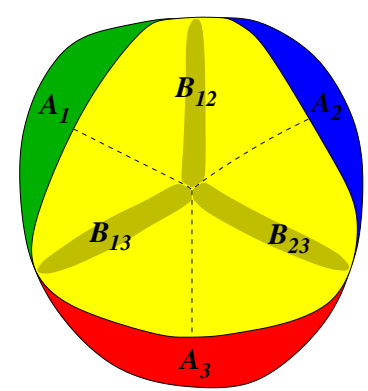

Figure 2: Illustration of feathering problem in $N$-view case. The figure shows the visible regions for three views. The central overlap region is partitioned into three regions separated by dashed lines, based on the two views which minimise the quantity $d\left(\boldsymbol{p}, \boldsymbol{A}_{k}\right) / s_{k}$. The feather within each such region is represented with a darker shade of yellow.

The decomposition into a sequence of pairwise image blends presents the advantage that at each pixel, at most two views contribute, thus eliminating excessive blur due to blending a large number of views in the same overlap region. From an implementation point of view, using a pairwise blending strategy reduces the overhead due to the increase in the number of views. A potential limitation of the decomposition into pairwise image blends is that we must ensure that all regions connect without producing any visible seam. In practice this is usually the case. A similar type of algorithm has been used by Gracias et al. in [9] in the context of image mosaicking.

\section{Overview of the rendering pipeline}

We now describe a full rendering pipeline which incorporates the dynamic feathering algorithm. The system is implemented in $\mathrm{C}++$ using the OpenGL API [1]. The OpenGL Shading Language is used to implement some of the blending operations directly in the fragment shader. The implementation uses hardware acceleration at most stages of the rendering 
process, with the exception of some parts of the dynamic feathering algorithm which are done in the CPU. Pseudo-code describing the rendering pipeline is given in Algorithm 4. The implementation of each module is detailed in the next section.

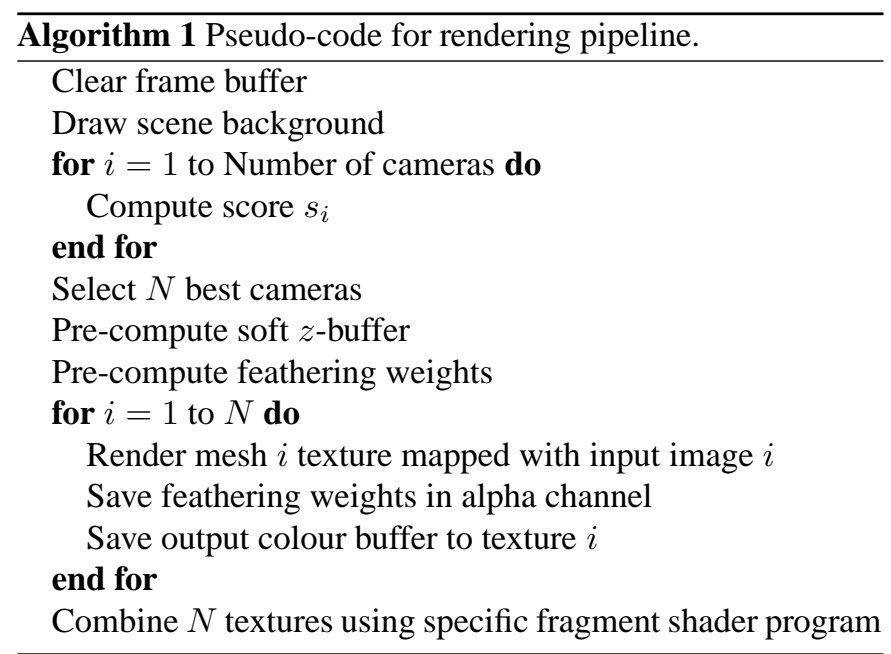

\section{Implementation details}

We detail the critical parts of the algorithm described in Section 4 focusing on practical implementation. In particular, more detail is given on the implementation of a fast dynamic feathering algorithm.

\subsection{Camera selection algorithm}

Scores are assigned to each input camera by computing the inverse of the angle between their optical axis and the optical axis of the rendering camera. This is a simple strategy which typically works well for scenes where there are no extreme variations in zoom factors. More sophisticated selection strategies incorporating distance between image centres (see $[6,13])$, camera resolution and field-of-view $[2,21]$ could also be considered. This was not necessary for the type of scene considered in this paper.

For a given viewpoint, we select the $k$-nearest cameras with $k$ being typically equal to 2 or 3 . In principle, the algorithm can operate with an arbitrary number of views. However in practice we found it desirable to try to keep the number of blended views to a small number. In particular, in the case of inaccurately calibrated data, such as for the scene we consider, blending with a larger number of views becomes detrimental to the rendering quality. Another practical motivation for using a small number of blended images is to keep the complexity of the algorithm to a minimum and achieve higher rendering frame rates.

\subsection{Mesh rendering with a soft $z$-buffer}

For each input camera, the geometry is represented as a triangular mesh textured with the input image. $z$-buffering is the conventional way to compute visibility in a rendering pipeline. Such an algorithm proceeds by storing the closest depth value at each pixel in a buffer and accepts only incoming primitives which are located at a lower depth than the one already stored. In the case of our view-dependent geometry representation which consists of non-exactly overlapping meshes, such a $z$-buffering algorithm would result in a noisy appearance with patches coming from different images, depending on which mesh is overlapping the others at each point. This phenomenon is known as z-fighting or bleeding in the computer graphics literature.

Smooth blending of closely overlapping regions is achieved by introducing a tolerance in the $z$-buffer visibility test. This approach is called soft $z$-buffer in [17] or $\epsilon$ - $z$-buffer in [13]. Such rendering is done in two passes. During the first pass, meshes are rendered successively with conventional $z$-buffering. During this pass, for efficiency, meshes can be rendered unlit, untextured, flat-shaded, and the colour buffer is made non writable. At the end of this pass, the $z$-buffer contains the closest depth value at each pixel. During the second pass, each mesh is rendered textured to the colour buffer with a small positive offset $\epsilon$ added to the $z$-buffer which is made read-only. Pixel dependent weights are stored in each image's alpha channel.

\subsection{Dynamic feather computation}

The pseudo-code for the dynamic feathering algorithm is given in Algorithm 5.3. The algorithm can be broken down into three main stages. The first stage, implemented in the GPU, consists in computing the visible regions for each view. This is done by drawing successively the different views, each assigned with a distinctive colour, into an auxiliary buffer. At each pixel, visibility for a given view is stored in an assigned bit of the auxiliary buffer. For example, with three views, a simple assignment would consist in using a different colour channel for each view. If an eight bit per channel colour format is used, up to 24 different views could be stored. This is more than the number we would expect to combine for a given view. With each view assigned a given colour code, all views are rendered successively as unlit, untextured, flatshaded meshes coloured with their assigned colour. Blending is enabled and the blending function set to additive so that at the end of this pass, pixels in the auxiliary buffer contain a colour encoded visibility representation. The second stage consists in computing the distance $d\left(\boldsymbol{p}, \boldsymbol{A}_{i}\right)$ at each pixel of the view to render. We use the Chamfer distance transform [3] to compute accurately this set of distances at a low computational cost. The algorithm requires only two passes in the auxiliary image computed at the previous stage. While computing these distances, the indices of the foreground pixels are stored in a list which is used for fast access to these pixels at the next stage. The last stage consists in assigning the weights. Contributing views at this pixel are determined based on the colour code stored in the auxiliary buffer. If a single view contributes at this pixel, the weight is set to 1 for this view and 0 for all others. If multiple views overlap at this pixel, the two views which minimise $d\left(\boldsymbol{p}, \boldsymbol{A}_{k}\right) / s_{k}$ are selected and blending weights are computed according to Eq. (4). 


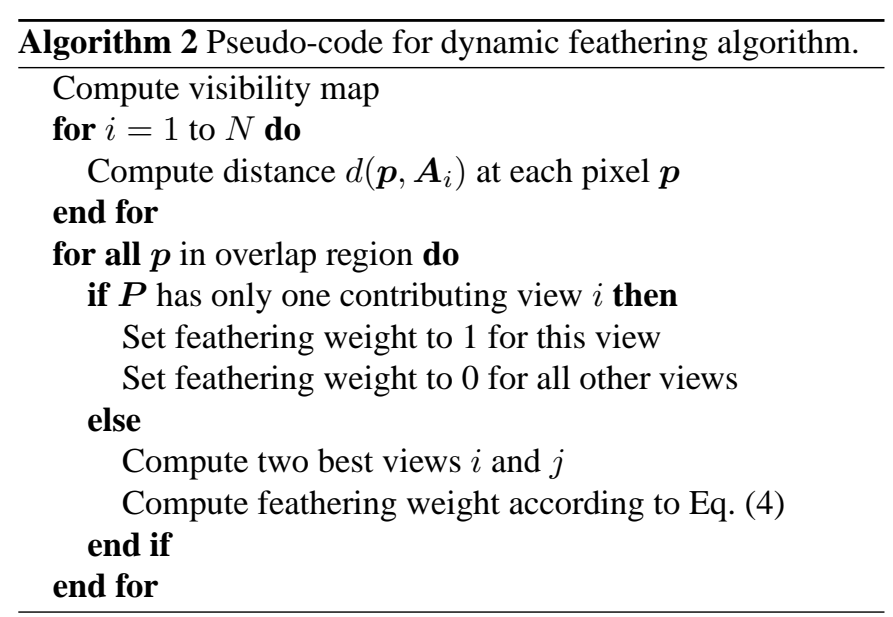

These last two stages are currently implemented in the CPU and are the parts of the algorithm which limit its rendering speed. Future work will concentrate on implementing a GPUbased distance transform, which should considerably speed up the algorithm. Feathering weights are stored in a texture map for each view used for blending. These weights are then combined with other per pixel blending weights stored in the alpha channel of each rendered view by enabling alpha blending, and rendering them on a screen size quad. The per pixel blending weights could correspond to surface sampling weights as in [17], or per pixel quality measures as in [13]. In our implementation we weight each pixel with its opacity value which was pre-computed by a matting algorithm [11].

From an implementation point of view, we observed that often there exist isolated pixels at object boundaries which are visible in a single image. Such pixels affect the computation of the distance maps and introduce noise during feather computation. We found in practice that better results are obtained by applying one pass of the open morphological operator [7] in the image in order to eliminate such isolated pixels. This usually results in cleaner feathers.

\subsection{Blending of separate image contributions}

Blending of the different views requires computing a weighted average of the different contributing images. We carry out this operation by multi-texturing the separate contributions onto a screen size quad. Addition and normalisation of the blended contributions is performed directly in the fragment shader.

\section{Results}

\subsection{Synthetic data}

We consider a simple example of a synthetic sphere textured with a checker pattern. Three views separated by a $45^{\circ}$ baseline were generated (see Fig. 3). In order to model errors in camera calibration, the coordinates of the image centre were shifted by an arbitrary 3 pixel offset for each camera, which results in a small image misalignment.

In a first experiment, we used only the first two views to synthesise an interpolated view of the sphere located half way between the two input cameras. Four different techniques are considered: "no feather", "boundary feather", "sharp feather" and "dynamic feather". The results obtained are shown in Fig. 4. For each technique, we show the composite image as well as the feathering weights which are represented with false colours. In this representation, each colour channel represents a weight for a given image. The first technique referred to as "no feather", consists in blending over the full overlap region without feathering. This results in a large amount of image blur and also ghosting artefacts such as double edges. In addition, discontinuities are present at boundaries between regions textured using different input images. The second technique, referred to as "boundary feather" attenuates the weights at each image boundary using a ramp style transition. This eliminates the discontinuities which were affecting the previous method, however it still results in a large amount of blur as well as double edges. The third and the fourth techniques are based on the dynamic feathering algorithm proposed. In the case of the "sharp feather" method, the dynamic feathering algorithm was used to compute a feather with zero width. This produces a hard segmentation of the images into two regions separated by a visible discontinuity. Finally the last method uses the dynamic feathering algorithm with a larger feather width. It can be observed that the amount of blur is now significantly reduced compared to the first two methods, while still producing smooth transitions with no visible seam. Fig. 5 shows a transition between the viewpoints of the first two images using the dynamic feathering algorithm. Note how the feather smoothly slides from one side of the sphere to the other as the camera viewpoint changes.

In a second experiment, the same experiment is repeated but this time with all three views. Fig. 6 shows the synthesis of a novel viewpoint centred at a point approximately equidistant from the three input cameras. The two techniques which apply either no feathering or only a boundary feather result in a large blurring area. In fact, due to the increased number of blended views, the amount of blur has increased in comparison with the two view case. The dynamic feathering algorithm on the other hand produces smooth views with considerably less image blur. An example of transition between two views is shown in Fig. 7.

\subsection{Real data}

We now present results on images of a football game. The scene was captured with 15 standard definition cameras covering approximately half of the football pitch. This example was chosen explicitly because camera calibration data is particularly bad. Dense depth maps of the scene were generated using our dense stereo reconstruction which uses graph-cuts [10]. Reconstruction is inaccurate due to camera calibration errors. Two examples of captured views are shown in Fig. 8. Opacity values estimated with the matting algorithm described in [11] provide additional pixel dependent weighting.

The results are shown in Fig. 9. Due to space limitations 

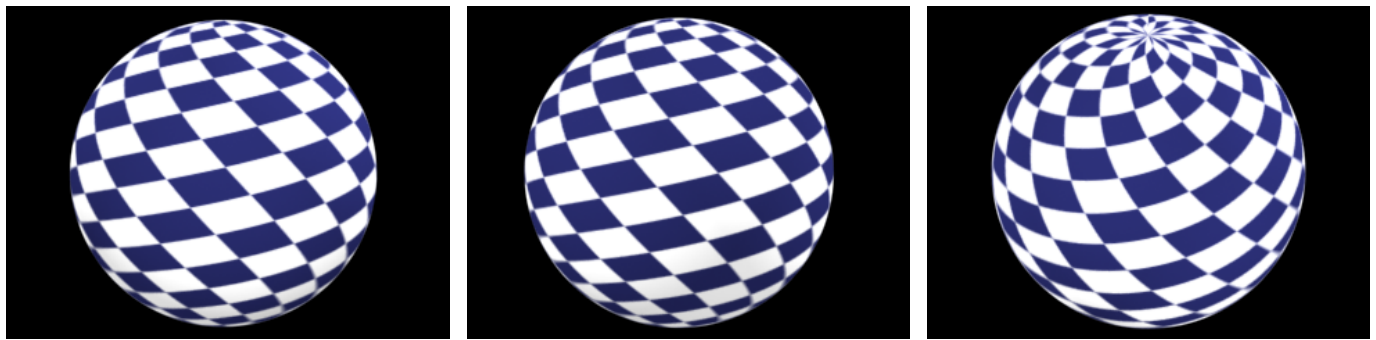

Figure 3: Three synthetic images of a sphere textured with a checker pattern.

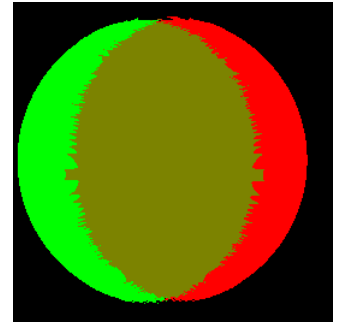

(a) No feather

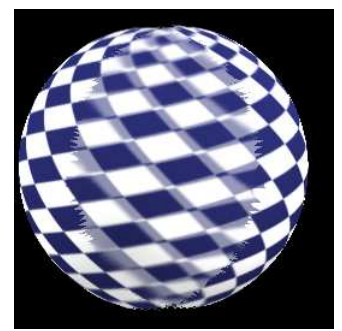

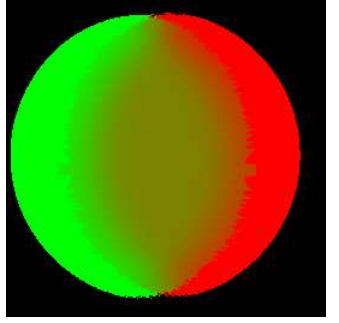

(b) Boundary feather

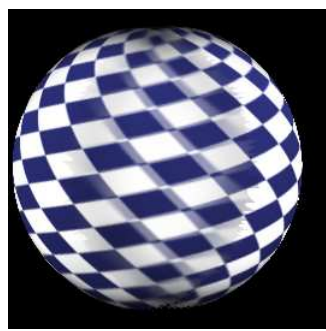

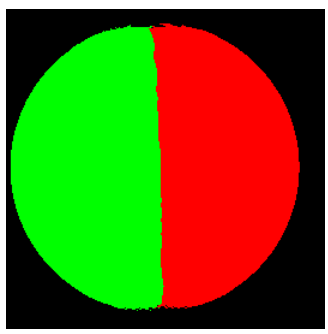

(c) Sharp feather

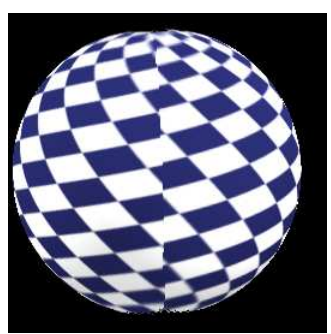

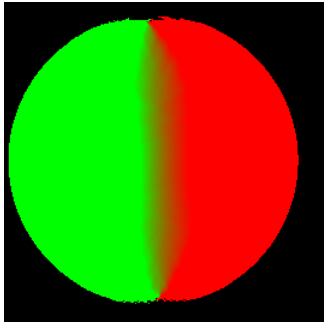

(d) Dynamic feather

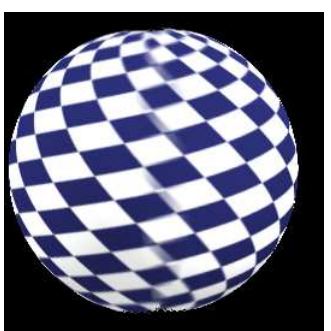

Figure 4: Rendering of an interpolated view located half way in between the first two images shown in Fig. 3. The rendered view is texture mapped using only these two input images. The top row shows the blending weights in false-colours. The bottom row shows the rendered image. The four columns correspond to four different feathering techniques.
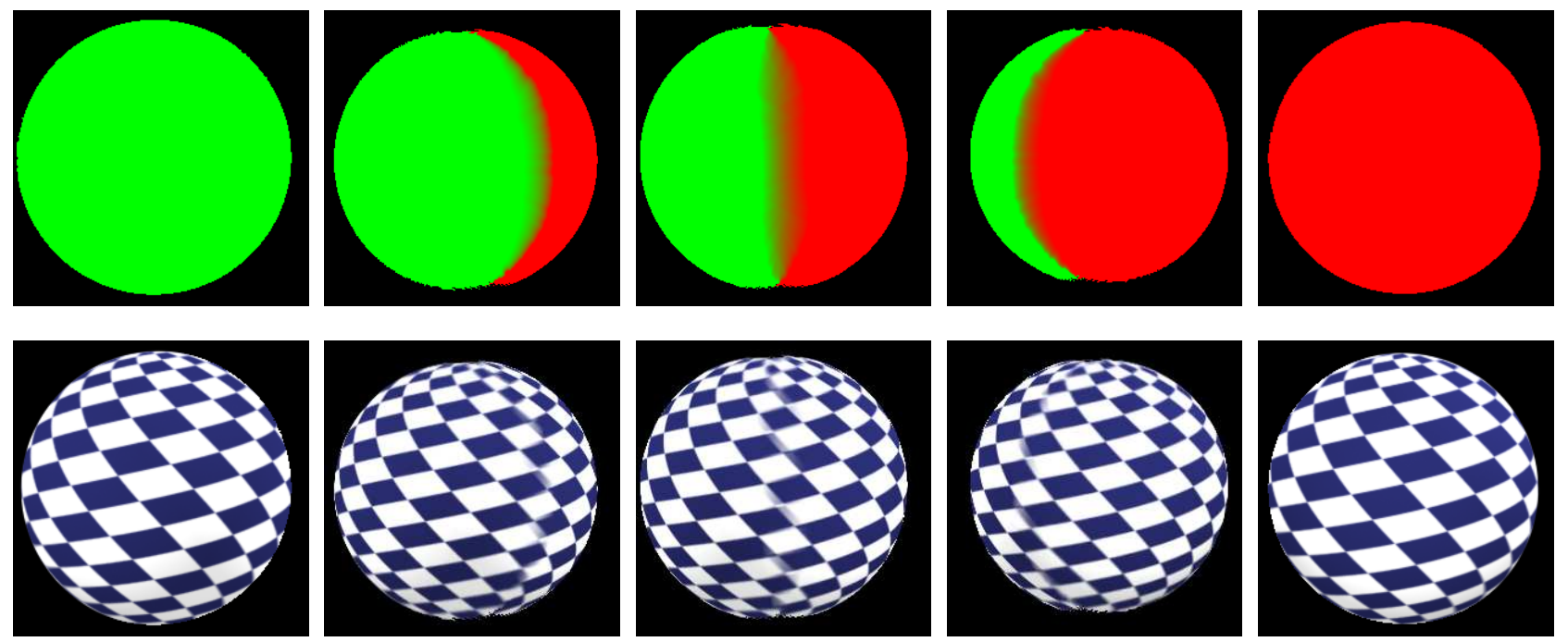

Figure 5: Rendering of a transition sequence from the first image to the second image shown in Fig. 3 using the dynamic feathering technique. The rendered views are texture mapped using only these two input images. The top row shows the blending weights in false-colours. The bottom row shows the rendered image. 


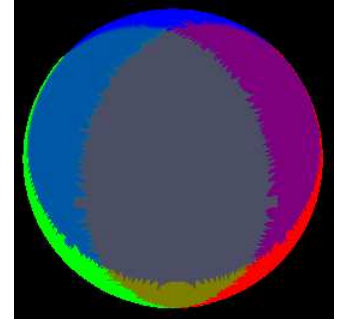

(a) No feather

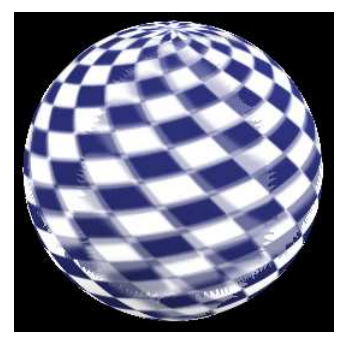

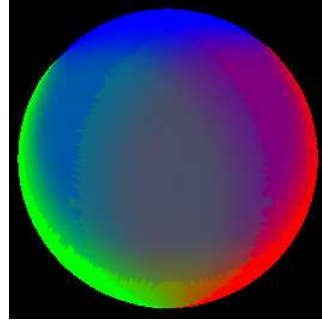

(b) Boundary feather

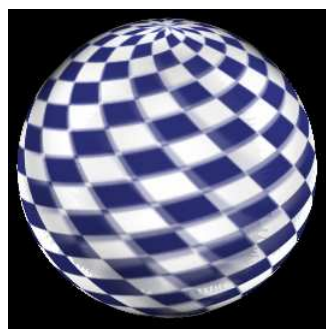

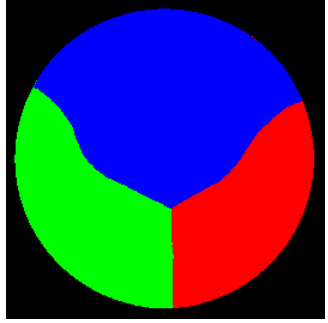

(c) Sharp feather

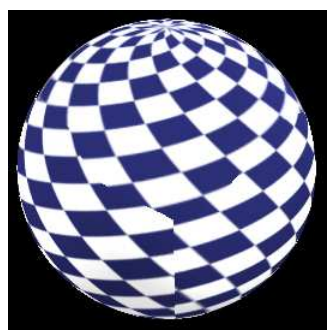

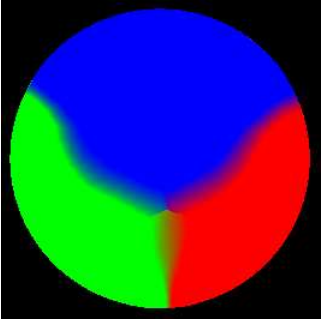

(d) Dynamic feather

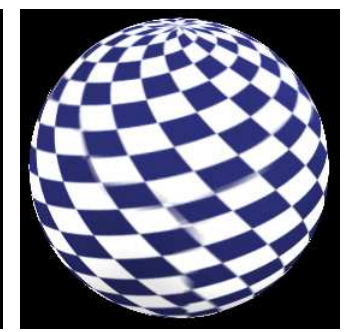

Figure 6: Rendering of an interpolated view from a viewpoint approximately equidistant from the three viewpoints used to render the input images shown in Fig. 3. The rendered view is texture mapped using all three input images. The top row shows the blending weights in false-colours. The bottom row shows the rendered image. The four columns correspond to four different feathering techniques.

and the larger image dimensions, results are shown for only two techniques: "no feather" and "dynamic feather". It is noticeable on the false-colour images that there is a large proportion of overlap between textures if no feather is applied. This is not visible in most areas of the appearance images because of the uniform scene colours, however this becomes more visible at textured areas such as players numbers which usually appear crisper with the dynamic feathering algorithm.

Note that due to small image player size, the improvement obtained with the dynamic feathering algorithm is not as large as we could have expected. This is partly due to fact that the resolution of our input images is low (dimension 720 by 288) and also to the type of scene we considered. We expect to obtain larger improvements on scenes where there is a larger number of pixel overlap. In particular we anticipate that the feathering method will become more advantageous with new captures using high definition images and also for different types of scenes where the foreground occupies a larger portion of the image.

Finally, we illustrate the application of the dynamic feathering algorithm for free-viewpoint video by showing examples of sequences of images synthesised with this technique in the case of a fixed viewpoint (Fig. 10) and also in the case of a fly-around at a frozen time instant (Fig. 11). The real scene considered contains 15 meshes each made of approximately 50,000 triangles, and renders at an interactive frame rate of 5 frames per second for an output resolution of 800 by 600 pixels on a $3 \mathrm{GHz}$ Pentium Xeon PC equipped with a GeForce 7600GS graphics card.

\section{Conclusions and future work}

We presented a hybrid view-dependent rendering technique for compositing multiple views in the presence of inaccurate geometry or camera calibration. The technique operates by computing, at interactive frame rate, a view-dependent blending region that reduces image blur while preserving smooth spatial and temporal transitions. The improvements achieved by applying the technique were demonstrated on synthetic and also real imagery with large camera calibration and geometric errors.

In future work, we would like to work on optimising the code for real-time rendering. Our code is still highly unoptimised and we fully expect to improve the rendering speed in future implementations. In particular, we would like to implement in the GPU the distance map computation, which currently dominates the algorithm run-time. Another possible avenue for future work would be to investigate more sophisticated algorithms for optimising feather position. In particular, we would like to investigate the effects of using image gradient information to generate seams within uniform regions and thereby reduce blurring and double exposure artefacts.

\section{Acknowledgements}

This work was supported by the DTI Technology Programme project 'iview: Free-viewpoint Video for Interactive Production' TP/3/DSM/6/I/15515 and EPSRC Grant EP/D033926/1. The authors gratefully acknowledge the project partners for providing the sports footage and discussion of the research reported in this paper. 

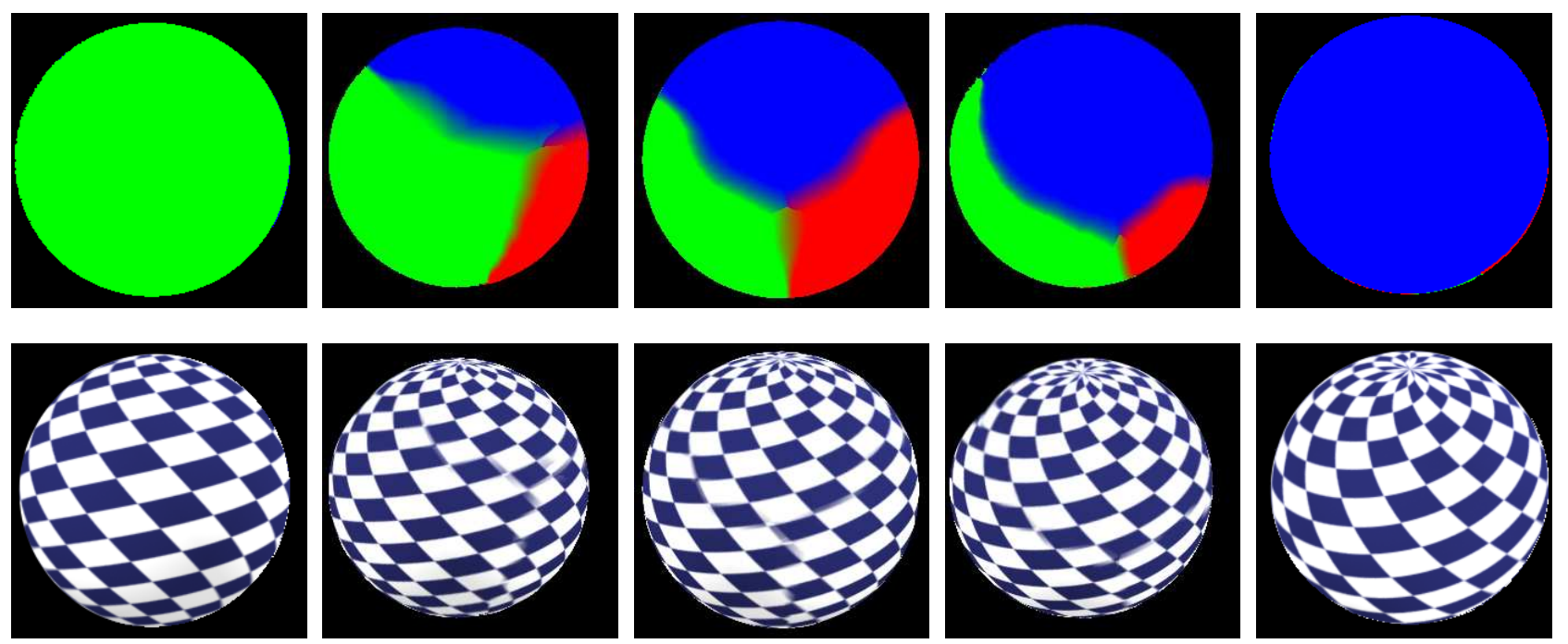

Figure 7: Rendering of a transition sequence starting at the first view and finishing at the last view shown in Fig. 3 using the dynamic feathering technique. The rendered view are texture mapped using all three input images. The top row shows the blending weights in false-colours. The bottom row shows the rendered image.

\section{References}

[1] http://www.opengl.org/.

[2] C. Buehler, M. Bosse, L. McMillan, S. Gortler, and M. Cohen. Unstructured lumigraph rendering. In SIGGRAPH, pages 425432, 2001.

[3] M.A. Butt and P. Maragos. Optimum design of chamfer distance transforms. In IEEE Transactions on Pattern Analysis and Machine Intelligence, pages 1477-1484, 1998.

[4] P. Debevec, Y. Yu, and G. Boshokov. Efficient view-dependent image-based rendering with projective texture-mapping. In Eurographics Rendering Workshop, 1998.

[5] P.E. Debevec, C.J. Taylor, and J. Malik. Modeling and rendering architecture from photographs: a hybrid geometry- and imagebased approach. In SIGGRAPH, pages 11-20, 1996.

[6] J.-F. Evers-Senne and R. Koch. Image based interactive rendering with view dependent geometry. EUROGRAPHICS, 22(3):573-582, 2003.

[7] R. C. Gonzalez and R. E. Woods. Digital Image Processing. Addison-Wesley, 1992.

[8] S.J. Gortler, R. Grzeszczuk, R. Szeliski, and M.F. Cohen. The lumigraph. In SIGGRAPH, pages 43-54, 1996.

[9] N. Gracias, A. Gleason, S. Negahdaripour, and M. Mahoor. Fast image blending using watersheds and graph cuts. In Proc. British Machine Vision Conference, volume II, pages 469-478, 2006.

[10] J.-Y. Guillemaut, A. Hilton, J. Starck, J. Kilner, and O. Grau. A Bayesian framework for simultaneous matting and 3D reconstruction. In International Conference on 3-D Digital Imaging and Modeling, pages 167-174, August 2007.

[11] A. Levin, D. Lischinski, and Y. Weiss. A closed form solution to natural image matting. In Proc. IEEE Conference on Computer Vision and Pattern Recognition, pages 61-68, 2006.

[12] M. Levoy and P. Hanrahan. Light field rendering. In SIGGRAPH, pages 31-42, 1996.
[13] R. Pajarola, M. Sainz, and Y. Meng. DMesh: Fast depthimage meshing and warping. International Journal of Image and Graphics, 4(4):1-29, 2004.

[14] P. Pérez, M. Gangnet, and A. Blake. Poisson image editing. In SIGGRAPH, pages 313-318, 2003.

[15] D. Porquet and J.-M. Dischler. Real-time per-pixel viewpoint interpolation. In Theory and Practice of Computer Graphics (TPCG'04), pages 219-225, 2004.

[16] D. Porquet, J.-M. Dischler, and D. Ghazanfarpour. Real-time high-quality view-dependent texture mapping using per-pixel visibility. In Proc. GRAPHITE 2005, pages 213-220, 2005.

[17] K. Pulli, M. Cohen, T. Duchamp, H. Hoppe, L. G. Shapiro, and W. Stuetzle. View-base rendering: Visualizing real objects from scanned range and color data. In Eurographics Workshop on Rendering Techniques, pages 23-34, 1997.

[18] R. Raskar and K.-L. Low. Blending multiple views. In Pacific Conference on Computer Graphics and Applications, pages 145-153, 2002.

[19] H. Y. Shum and S. B. Kang. A review of image-based rendering techniques. In IEEE/SPIE Visual Communications and Image Processing (VCIP), pages 2-13, 2000.

[20] R. Szeliski. Image alignment and stitching: a tutorial. Foundations and Trends in Computer Graphics and Vision, 2(1):1-104, December 2006.

[21] F. Verbiest, G. Willems, and L. Van Gool. Image-based rendering for photo-realistic visualization. Virtual and Physical Prototyping, 1(1):19-30, 2006.

[22] G. Willems, F. Verbiest, M. Vergauwen, and L. Van Gool. Real-time image based rendering from uncalibrated images. In International Conference on 3-D Digital Imaging and Modeling, pages 221-228, 2005.

[23] C. Zhang and T. Chen. A survey on image-based rendering: Representation, sampling and compression. Signal Processing: Image Communication, 19(1):1-28, 2004. 

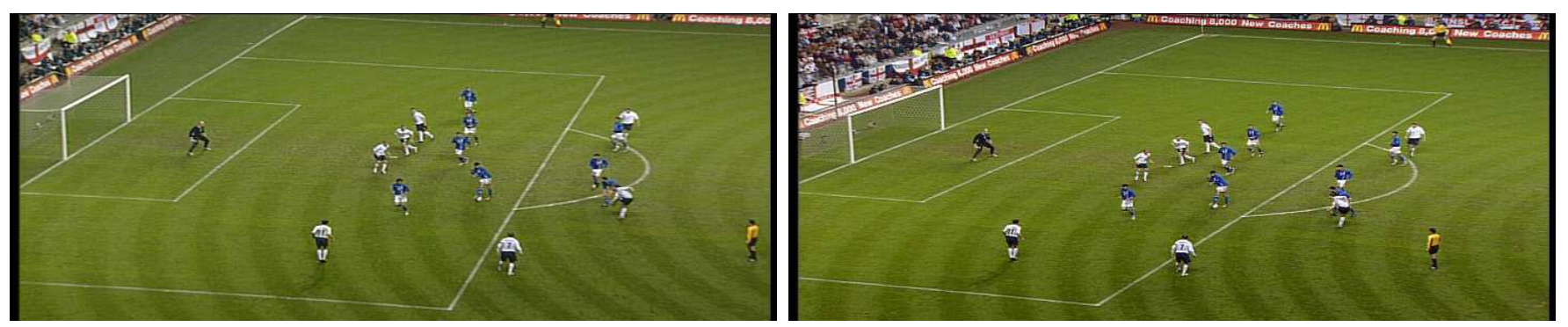

Figure 8: A pair of input images from the 15 camera capture of a football game.

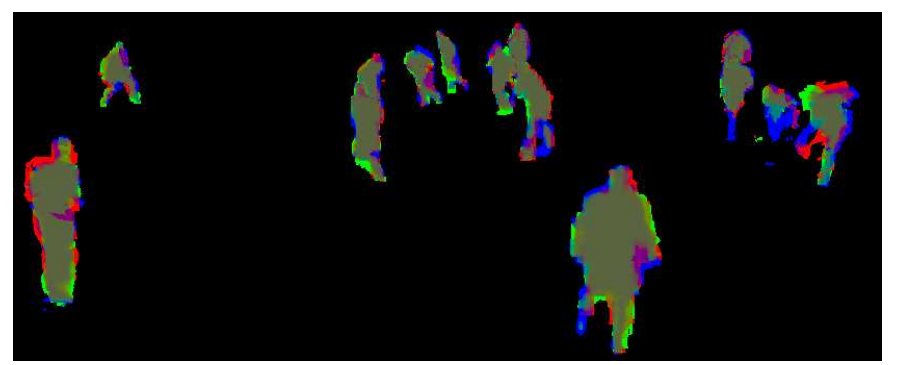

(a) No feather

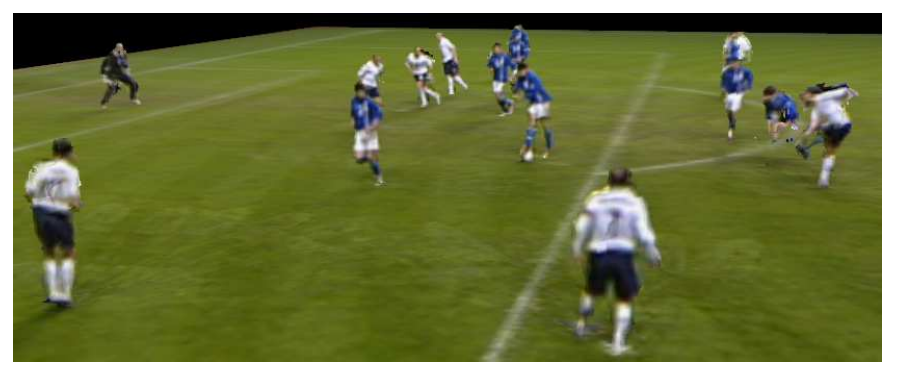

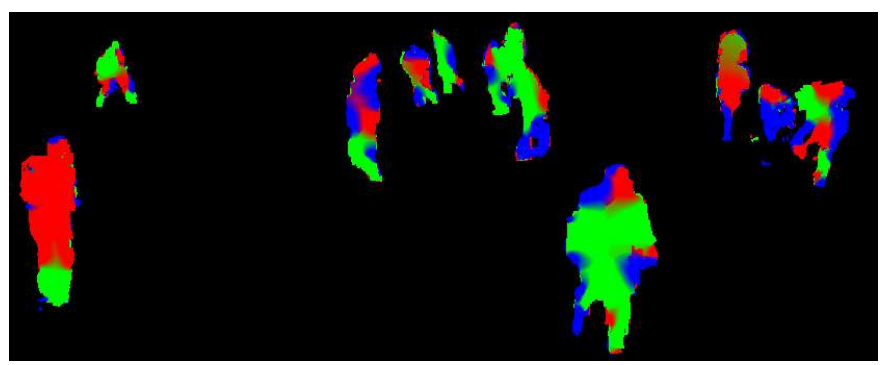

(b) Dynamic feather

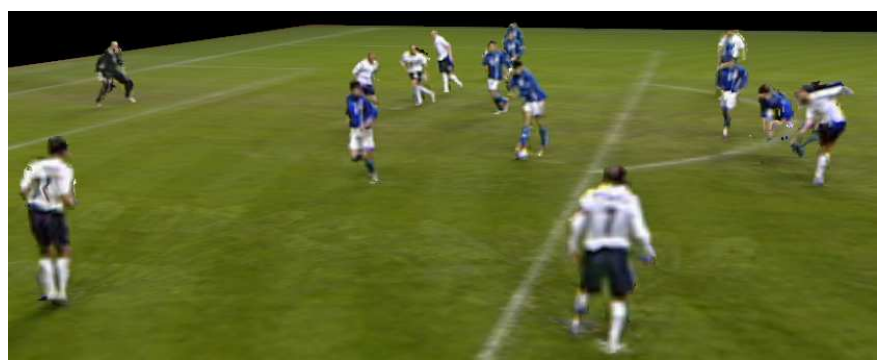

Figure 9: Rendering of a novel view-point located in between but well below the cameras used to capture the two images shown in Fig. 8. The top row shows the blending weights in false-colours. The bottom row shows the rendered image. The two columns correspond to two different feathering techniques.
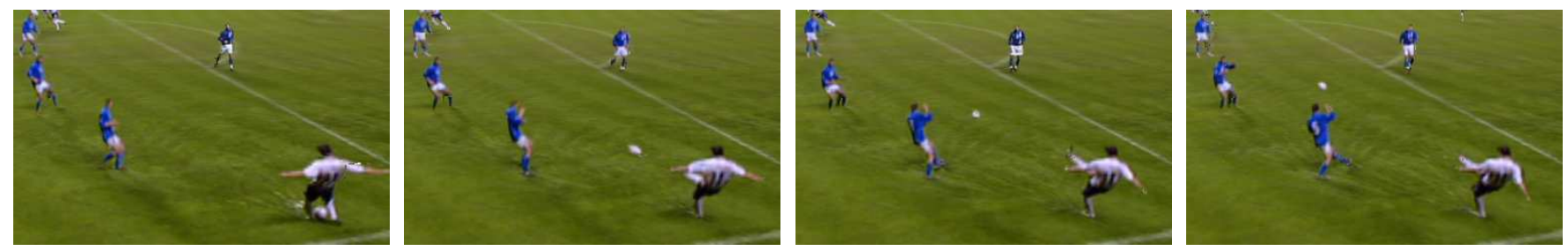

Figure 10: A few images from a sequence rendered from a fixed interpolated viewpoint using the dynamic feathering algorithm.
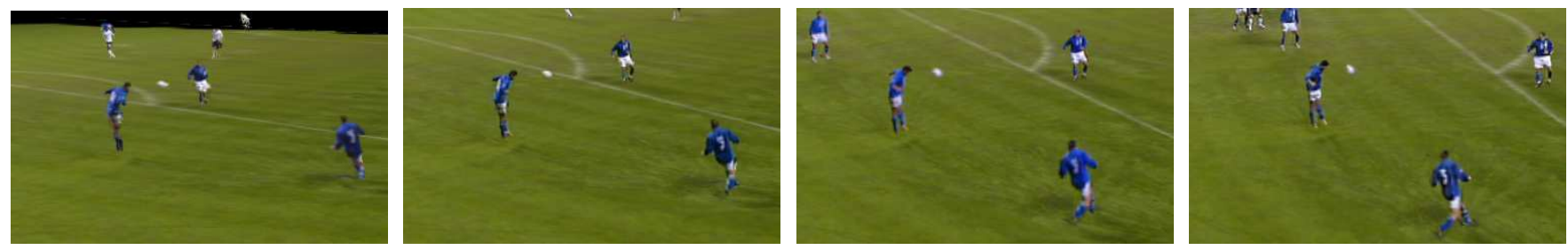

Figure 11: A few images from a fly-around sequence with frozen time using the dynamic feathering algorithm. 